\title{
Ocular dynamics of Garcinia cola (Heckel) on healthy volunteers following bolus ingestion
}

\section{SA Igwea, DN Akunyilib, PC Igwe ${ }^{c}$ and EU Ikonne}

a Department of Pharmacology and Therapeutics, College of Health Sciences, Ebonyi State

University Teaching Hospital, Abakaliki, Nigeria

b Department of Pharmacology and Therapeutics, College of Medicine, University of Nigeria

Teaching Hospital, Enugu, Nigeria

c Department of Optometry, College of Medicine and Health Sciences, Abia State University, PMB 2000, Uturu, Nigeria

\section{Author for correspondence: <nwosuigwe@yahoo.com>}

\begin{abstract}
Garcinia cola Heckel, an angiospermae belonging to the family Guttiferae, is known in commerce as bitter cola. It is a plant found in the West African subregion, mostly in moist conditions, and often in association with Cola acuminata. The seeds are highly valued ingredients in African ethonomedicine. The seeds have several social uses and applications in folk medicine. These seeds are ordinarily chewed by the local people without prescription or restriction. In an in vivo case control study, the effects of Garcinia cola on some visual functions: pupil diameter, near point of convergence (NPC), amplitude of accommodation (AA), intraocular pressure (IOP), visual acuity (VA) and habitual phoria; following bolus ingestion of $20 \mathrm{~g}$ was undertaken using healthy visually active volunteers. Results showed that bolus ingestion of Garcinia cola constricted the pupil by $68 \%$, reduced the NPC by $28 \%$, increased the AA by $17.8 \%$ at peak effect and decreased the intraocular pressure by $31 \%$ without affecting the distance and near VA. The lateral phoria at far and near tended towards esophoria. Possibly the miotic effect and reduction in IOP could be of benefit to patients with raised IOP where conventional drugs may not be effective. Also it is possible that the effects on phoria could be exploited in oculomotor function in combination with visual training.
\end{abstract}

Keywords: Bolus ingestion, Garcinia cola, ocular dynamics, oculomotor, intraocular pressure.

\section{Introduction}

Earlier reports have shown that edible plants and plant materials have nutritional and medicinal values as well as, adverse effects ${ }^{1}$. One such plant is Gercinia cola Heckel, family Guttiferae commonly known in commerce as bitter cola and found in the West African sub-region mostly, in moist conditions, and often in association with Cola acuminata. The seeds are the most important products and are found in markets from Sierra Leone to Southern Nigeria. G. cola is an angiospermae belonging to the family Guttiferae with a voucher number EFH 3056 (Enugu Forestry Herbarium) and authenticated by Prof JC Okafor of Enugu State University of Science and Technology, Ebeano City, Enugu.

On chewing, G. cola seeds have a bitter astringent and resinous taste, somewhat resembling that of raw coffee, followed by a slight sweetness. They are appreciated rather as an adjuvant than a substitute for true cola thereby increasing the users enjoyment of larger quantities without disposition. Garcinia cola (bitter cola) increases the flavour of native liquor and the residue after chewing is white.

Bitter cola is highly valued ingredient in African ethnomedicine because of its varied and numerous uses which are social and medicinal thus making the plant an essential ingredient in folk medicine. 
G. cola is indicated in the treatment of head and chest cold, colic, improves singing voices through soothing effect on vocal cords, relieves coughs and hoarseness of voice ${ }^{2}$. The fresh seed is also chewed as an aphrodisiac while the dried seeds are used in the treatment of dysentery and diarrhoea. Further indications for Garcinia cola include postpartum hemorrhage, urinary tract infection, emesis and poisoning due to Strophantus gratis and gonorrheal orchitis ${ }^{3}$ and quite recently the water extracts of $G$. cola have been shown to possess anti fungal activities on purulent human ocular discharges ${ }^{4}$.

The masticated fruit pulp has been used as an oral antiseptic and in the treatment of cuts and sore throat ${ }^{5}$. The roots and stem which serve as bitter chewing sticks are also used for oral hygiene. The bark can be powdered and used in treating malignant tumors, pyrexia as well as serve as bitter tonic and astringents ${ }^{5}$. The latex obtained from the stem bark has been reported to be effective against parasitic skin diseases when applied topically for wound dressing ${ }^{6}$.

G. cola fruits are also used as anti-arthritic agents while the seeds are used for the treatment of cough $^{7}$, liver disorder ${ }^{8}$ and as a possible substitute for hop in the Beer industry9.

G. cola contains a wide spectrum of organic compounds such as flavonoids which confer on it some anti-microbial and anti fungal actions against gram negative and gram positive microorganisms ${ }^{10}$. Earlier in 1970, Harley $^{3}$ had reported the anti-tusive, anti-tumour and aphrodisiac activities of $G$. cola while the flavonoids extracted from the seeds have been effective in the treatment of capillary fragility, retinal hemorrhage in hypertension, diabetic retinopathy, purpura, rheumatic arthritis, radiation disease, habitual abortion, frostbite, anaphylactic shock, experimental cancer and in the prevention of chromodacroyorrhoea produced by dietary and environmental stress ${ }^{11}$. In 1985, Elekwa ${ }^{12}$ also reported the prevention of thioacetamideinduced hepatotoxicity in rats by $G$. cola extracts.

In spite of the varied use and effects of $G$. cola in ethnomedicine, no attempt has been made to document its effect on visual functions. The purpose of this case control study is therefore to determine the effects of $G$. cola on pupil size, near point of convergence, visual acuity, amplitude of accommodation, intraocular pres- sure and lateral phoria.

\section{Materials and Method}

This was an in vivo case control study in which young people attending the Abia State University Optometry Clinic, Uturu were enlisted into the study who also served as their own internal control. The initial or preingestion values of these visual functions served as internal control for each volunteer. The use of placebo for the study would have introduced complications.

After the screening exercise by the optometrists, involving ocular health ophthalmoscopy, biomicroscopy, pupillary light reflex, et cetera, twenty (10 males and 10 females) healthy young adults without any refractive errors, weighing between 55 and $62 \mathrm{~kg}$ (mean 61.25+ $2.25 \mathrm{~kg}$ ) were selected. The nature of the study was explained to them and their informed verbal/written consent obtained. The study was approved by the Ethical Committee of the Optometrists and Dispensing Opticians Board of Nigeria, as well as that of the University.

Prior to administering $G$. cola baseline values of the following visual functions were measured on all subjects: pupil size or diameter, near point of convergence, visual acuity, amplitude of accommodation, intraocular pressure and phorias, so that each subject would serve as his or her own internal control. The protocol of the study was further explained to the participants and then $20 \mathrm{~g}$ of $G$. cola was administered to each of the subjects, which was chewed and swallowed as bolus. Finally, in order to complete the protocol, $50 \mathrm{ml}$ of table water was given to each participant to thoroughly wash the masticated substance down the gut. The subjects were thereafter engaged in normal reading or simple in door exercise such as playing cards, ludo games, et cetera, for the next two hours during which time subjects did not ingest any thing further.

Preliminary studies had shown that the effects of $G$. cola do not manifest on the ocular tissues until 45 min after ingestion. Thereafter each of the above visual functions were repeated at 15 min interval for the next 2 hours.

Differences between values obtained before the ingestion of Garcinia cola and values obtained after ingestion of the substance were taken as the effect of the substance on the par- 
ticular visual function. Data were expressed as the mean \pm SEM. The results were further subjected to statistical analysis using ANOVA.

\section{Measurements}

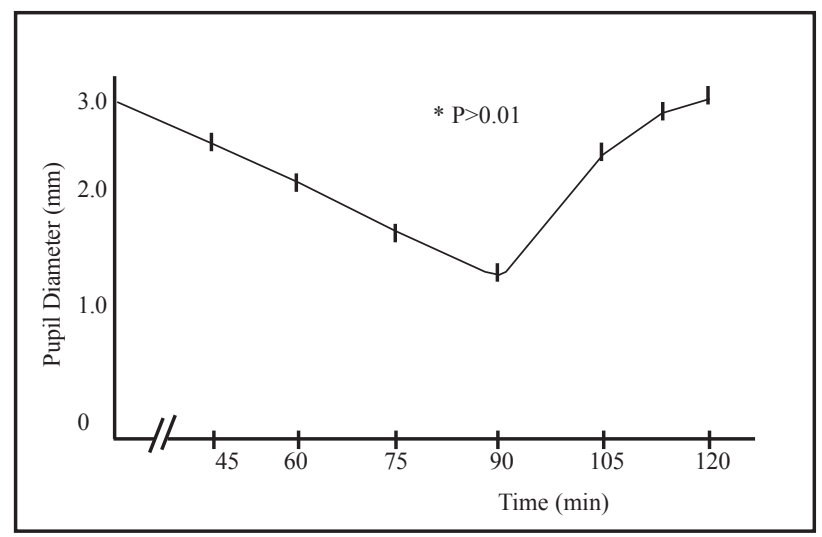

Figure 1: Mean changes in pupil size with time following the ingetion of $20 \mathrm{~g}$ of $G$. cola $(p>0.01)$. Each reading was taken 5 times $(n=5)$, per subject.

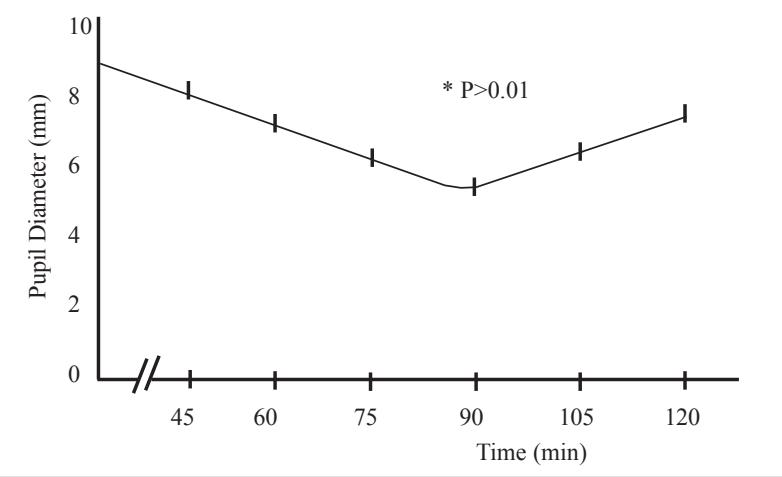

Figure 2: Mean changes in near point of convergence following the ingestion of $20 \mathrm{~g}$ of $G$. cola $(p>0.01)$. Each reading was taken 5 times $(n=5)$, per subject

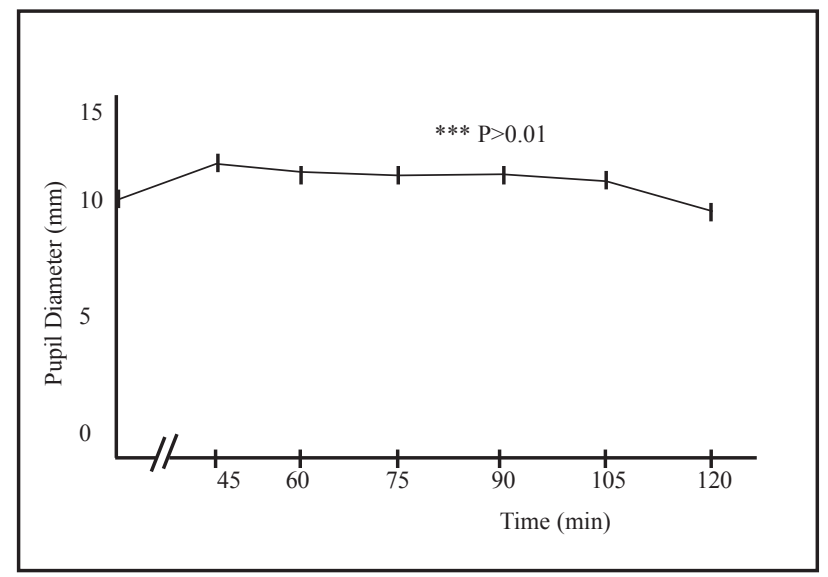

Figure 3: Mean changes in amplitude of accommodation following the ingestion of $20 \mathrm{~g}$ of $G$. cola $(p>0.01)$. Each reading was taken 5 times $(n=5)$, per subject a. Pupil diameter: This was measured using a pupillometer. The subject was asked to fixate straight ahead with the instrument placed below the lower lid and the value read directly from the device to the nearest tenth of a millimeter. This was done in three different positions and the average determined.

b. Near point of convergence (NPC): All the subjects had NPC of between 8 and $10 \mathrm{~cm}$ reference before the study. In the measurement of the NPC the examination room was fully illuminated. A pencil was held approximately $40 \mathrm{~cm}$ at the subject's eye level and the subject was asked to fixate at the tip of the pencil. The pencil was then adjusted slowly along the mid-line towards the midpoint between the subject's eye. The subject was instructed to follow the target inward with the eye as closely as possible and to report when the target doubled or broke into two. The point where the subject reported diplopia or when there was loss of a bifoveal fixation by the subject was taken as the near point of convergence and was measured with a meter-rule in $\mathrm{cm}$.

c. The distance and near visual acuity (VA) were recorded using the standard illuminated Snellen optotypes at the appropriate measuring distances $(0.4 \mathrm{~m}$ for near and 6 $\mathrm{m}$ for far respectively). The results are presented as means of five measurements.

d. The amplitude of accommodation (AA) was ascertained or measured using the negative lens to blur method at $33 \mathrm{~cm}$. Measurements were taken 5 times on each individual and averages determined.

e. The intraocular pressure (IOP) was measured using the Schiotz tonometer at 10:00 am, after anaesthesizing with $0.4 \%$ xylocaine solution, and with a weight of $5.5 \mathrm{~g}$ the value was read from the accompanying table. Average of 5 readings were taken and recorded in $\mathrm{mmHg}$.

f. The von Graeffe phoria was measured using the phoropter. The distance phoria was determined with the subject fixating a lineal letters on Snellen chart placed at $6 \mathrm{~m}$. Then a 15 pd base-in prism was introduced on the right eye while base-up introduced on the left eye. The chart appeared double. The $15 \mathrm{pd}$ base-in prism was gradually reduced 
Table 1: Distance phoria values in prism diopters, measured before ingestion of Garcinia cola and subsequently after 45 min and at 15 min interval for $2 \mathrm{hr}$ : These results are significant at $p>0.01$

\begin{tabular}{|c|c|c|c|c|c|c|c|}
\hline \multicolumn{8}{|c|}{ Phoria values (far) } \\
\hline $\mathrm{S} / \mathrm{No}$ & $\begin{array}{l}\text { Preingestion } \\
\text { value }\end{array}$ & $45 \mathrm{~min}$ & $60 \mathrm{~min}$ & $75 \mathrm{~min}$ & $90 \mathrm{~min}$ & $105 \mathrm{~min}$ & $120 \mathrm{~min}$ \\
\hline 1 & 1 eso & 2 eso & 3 eso & 3 eso & 3.5 eso & 2.6 eso & 1 eso \\
\hline 2 & ortho & 1 exo & 2 exo & 1 eso & ortho & 1 eso & ortho \\
\hline 3 & ortho & 1 eso & 2 eso & 2.5 exo & 3 eso & 2 eso & ortho \\
\hline 4 & ortho & 1 eso & 2 eso & 3 eso & 3.5 eso & 2 eso & ortho \\
\hline 5 & 1 exo & ortho & 1 eso & 2 eso & 2.5 eso & other & 1 exo \\
\hline 6 & 2 eso & 3 eso & 4 eso & 3 eso & 4.5 eso & 3 eso & 2 eso \\
\hline 7 & 1 eso & ortho & 1.5 eso & 2 eso & 2 eso & 1 exo & 1 exo \\
\hline 8 & 1 eso & 2 eso & ortho & 2 eso & 1 eso & 2 eso & 1 eso \\
\hline 9 & 1 eso & 2 eso & 3 esp & 3 eso & 4 eso & 2 eso & 1 eso \\
\hline 10 & ortho & 1 esp & 3 eso & 4 eso & 4 eso & 2 eso & ortho \\
\hline 11 & 1 eso & 1 exo & other & 1 eso & 2 eso & ortho & 1 exo \\
\hline 12 & ortho & 1 eso & 2 eso & 3 eso & 2 eso & 1 eso & ortho \\
\hline 13 & 1 eso & 2 eso & 1 esp & 3 eso & 3.5 eso & 2.6 eso & 1 eso \\
\hline 14 & ortho & 1 exo & 2 exo & 1 eso & ortho & 1 exo & ortho \\
\hline 15 & 1 exo & ortho & 1 eso & 2 eso & 2.5 eso & ortho & 1 exo \\
\hline 16 & 1 eso & 3 esp & 4 eso & 3 eso & 4.5 eso & 3 eso & 2 eso \\
\hline 17 & 1 exo & ortho & 1.5 eso & 2 eso & 2 eso & 1 exo & 1 exo \\
\hline 18 & 1 eso & 2 eso & ortho & 2 eso & 1 eso & 2 eso & 1 eso \\
\hline 19 & 1 exo & 1 exo & ortho & 1 eso & 2 eso & ortho & 1 eso \\
\hline 20 & 1 eso & 2 eso & ortho & 2 eso & 1 eso & 2 eso & 1 eso \\
\hline \multicolumn{8}{|c|}{$\begin{array}{l}\text { ortho }=\text { orthophoria } \\
\text { eso }=\text { esophoria } \\
\text { exo }=\text { exophoria }\end{array}$} \\
\hline
\end{tabular}

until there was vertical alignment of the chart. The amount of prism diopter obtained was recorded as the phoria value. For near phoria, the procedure was repeated at a distance of $40 \mathrm{~cm}$. Average of 5 measurements were used.

Garcinia cola seeds were supplied by a local farmer in Uturu so as to limit the effect of environmental variations on the seeds. The seeds were cleared of its outer covering, weighed and stored in separate dry plastic containers until required for use.

\section{Results}

a. Pupil Size: There was a reduction of the pupil size (diameter) from a mean initial value of $3.0 \pm 0.0 \mathrm{~mm}$ to a mean value of $1.0 \pm 0.1 \mathrm{~mm}$ at the peak effect, or $68 \%$ constriction. In Figure 1, there is a gradual miotic effect following bolus ingestion of the substance, until the $90^{\text {th }}$ minute when the constriction became more pronounced and highly significant $(p>0.01)$, but reversing gradually before the $120^{\text {th }}$ minute. This result is significant at $p>0.01$.

b. NPC: Each volunteer had a relatively normal NPC of between 8 and $10 \mathrm{~cm}$, and the mean NPC before the commencement of the study was $9.0 \pm 0.45 \mathrm{~cm}$. After ingestion of $G$. cola there was an improvement in the NPC and the mean value at the peak effect was $5.69 \pm 0.35 \mathrm{~cm}$ or $48 \%$ improvement or advancement. In Figure 2, the improvement in NPC was gradual and the advancement became highly significant at the $105^{\text {th }}$ minute ( $p>0.01)$ before reversing to the pre-experimental size.

c. VA: All the volunteers had normal VA at near (N5) and at far (6/6) respectively. These values remained the same following the ingestion of $20 \mathrm{~g}$ of Garcinia cola. Five different measurements were taken for each 
Table 2: Distance phoria values in prism diopters, measured before ingestion of Garcinia cola and subsequently after 45 min and at $15 \mathrm{~min}$ interval for $2 \mathrm{hr}$ : These results are significant at $p>0.01$

\begin{tabular}{|c|c|c|c|c|c|c|c|}
\hline \multicolumn{8}{|c|}{ Phoria values (far) } \\
\hline S/No & $\begin{array}{l}\text { Preingestion } \\
\text { value }\end{array}$ & $45 \mathrm{~min}$ & $60 \mathrm{~min}$ & $75 \mathrm{~min}$ & $90 \mathrm{~min}$ & $105 \mathrm{~min}$ & $120 \mathrm{~min}$ \\
\hline 1 & 4 exo & 3 exo & 2 exo & ortho & ortho & 3 exo & 4 exo \\
\hline 2 & 5 exo & 3 exo & 2 exo & 1 exo & 2 exo & 3 exo & 5 exo \\
\hline 3 & 8 exo & 6 exo & 4 exo & 2 exo & 3 exo & 5 exo & 8 exo \\
\hline 4 & 5 exo & 3 exo & 1 exo & ortho & ortho & 3 exo & 5 exo \\
\hline 5 & 6 exo & 5 exo & 4 exo & 2 exo & 3 exo & 5 exo & 6 exo \\
\hline 6 & 5 exo & 4 exo & 3 exo & 2 exo & 1 exo & 3 exo & 5 exo \\
\hline 7 & ortho & 3 eso & 2 eso & 2 eso & 4 eso & ortho & ortho \\
\hline 8 & 2 exo & 1 exo & 1 exo & ortho & ortho & 1 exo & 2 exo \\
\hline 9 & 5 exo & 3 exo & 1 exo & ortho & ortho & 3 eso & 5 eso \\
\hline 10 & 5 exo & 4 exo & 3 exo & 2 exo & 2.5 exo & 3 exo & 5 exo \\
\hline 11 & 6 exo & 5 exo & 4 exo & 10 exo & 4 exo & 4 exo & 6 exo \\
\hline 12 & 5 exo & 4 exo & 3 exo & 3 exo & 2 exo & 3 exo & 5 exo \\
\hline 13 & 8 exo & 8 exo & 9 exo & 2 exo & 10 ехо & 9 exo & 8 exo \\
\hline 14 & 6 exo & 5 exo & 4 exo & 10 exo & 4 exo & 4 exo & 6 exo \\
\hline 15 & 5 exo & 3 exo & 1 exo & ortho & ortho & 3 exo & 5 exo \\
\hline 16 & 4 exo & 3 exo & 2 eso & ortho & ortho & 3 exo & 4 exo \\
\hline 17 & 2 exo & 1 exo & 1 exo & ortho & ortho & 1 exo & 2 exo \\
\hline 18 & ortho & 3 eso & 2 eso & 2 eso & 4 eso & ortho & ortho \\
\hline 19 & 8 exo & 6 exo & 4 exo & 2 exo & 3 exo & 5 exo & 8 exo \\
\hline 20 & 4 exo & 4 exo & 3 exo & 2 exo & ortho & 3 exo & 4 exo \\
\hline \multicolumn{8}{|c|}{$\begin{array}{l}\text { ortho }=\text { orthophoria } \\
\text { eso }=\text { esophoria } \\
\text { exo }=\text { exophoria }\end{array}$} \\
\hline
\end{tabular}

individual.

d. AA: Garcinia cola increased the AA from an initial mean value of $9.70 \pm 0.33 \mathrm{D}$ to 11.38 $\pm 0.26 \mathrm{D}$, that is $17.8 \%$ increased at the peak effect. Figure 3 shows that the AA increased with time following bolus ingestion of the substance and the increase became significant at the $105^{\text {th }}$ minute $(p>0.01)$ before returning to pre-experimental value after 2 hours.

e. Intraocular pressure: The mean initial intraocular pressure (IOP) taken at 10:00 am local time was $22.48 \pm 1.22 \mathrm{mmHg}$ before the study and it dropped to $15.52 \pm 1.16$ $\mathrm{mmHg}$ at the peak effect following ingestion of $20 \mathrm{~g}$ of $G$. cola. When measurement was taken at the same time the following day the result showed a $31 \%$ reduction. The drop in IOP was significant at $p>0.01$.

f. The effects of G. cola on the phoria at far and near are shown in tables 1 and 2 respec- tively. The tables show that at the end of 2 $\mathrm{hr}$, post ingestion, the phoria had returned to pre-experimental values. These results are significant at $p>0.01$.

\section{Discussion and Conclusion}

According to the results obtained from this study, Garcinia cola, an angiospermae, produced a decrease in the pupil size and a drop in the intraocular pressure, caused improvements in the NPC, increased the AA while the lateral phoria at far tended towards esophoria and exophoria at near. The substance did not affect both the distance near VA. These results are time-dependent (see Figures 1, 2, and 3), and further demonstrate the miotic property of $G$. cola, which tends to support earlier work ${ }^{13}$. According to Igboko $^{13}$ aqueous extracts of $G$. cola contracted the smooth muscle of rabbit jejunum thus behaving like acetylcholine, which also produces miosis. This is suggestive of the action 
of $G$. cola on the parasympathetic system.

The effect on the ocular system can be so explained since $G$. cola by stimulating the parasympathetic system produces such effects as pupillary constriction (miosis), increase in the AA and improvement in the NPC, all miotic or parasympathomimetic effects.

Although the substance produced an increase in AA and NPC, and a decrease in the pupil size as well as the lateral phoria tending towards esophoria at far, but the strong association between accommodation and convergence $(r=0.96)$ and the steady phorias, the individual will maintain clear binocular vision after ingestion of G. cola.

However, decrease in pupil size accompanying the ingestion of bitter cola should affect the depth of focus. According to Green et al ${ }^{14}$, depth of focus is inversely proportional to the ocular focal length and the pupil size, and directly proportional to the just detectable retinal blur circles. The decrease in pupil size by $G$. cola would therefore affect both ametropes and emmetropes, care should be taken in making premature prescriptions to such subjects after ingestion of $G$. cola.

The local discovery of a drug that will lower the intraocular pressure will solve the problematic difficulties encountered in the management of glaucoma, a common cause of preventable blindness, more so, for cheaper purchase, availability and improved morbidity.

The constriction of the pupil has always been accompanied by increase in AA and NPC via the parasympathetic mechanism, with a consequent fall in IOP through the facilitation of aqueous outflow. This decrease in IOP obtained after ingestion of $G$. cola would be attributed to vasoconstrictor amines and $\mathrm{N}$-nitroso-N-methyl urea, a systemic hyperosmotic agent present in the seeds of $G$. cola ${ }^{15}$ which actually caused the decrease in the volume of aqueous humour. These results give evidence of ocular usefulness of G. cola and is obvious that more still needed to be done to isolating and characterizing the active constituents responsible for the ocular effects.

The change in AA, is two-way in that the resultant effect for a young adult may not be the same for elderly individual. A presbyopic or near presbyopic individual who after chewing G. cola had his AA increased will experience symptoms associated with presbyopia later than emmetrope. On the other hand, a young adult whose AA is normal or high will have the AA further increased after ingestion of $G$. cola with consequent synkinetic increase in convergence thus leading to convergence excess or divergence insufficiency with their associated symptoms. Though increase in AA and esophoria may be beneficial to the individual with divergence excess problems, one has to be cautious in its $(G$. cola) use because of the effect on the pupil size.

Finally, the effect on phoria can be exploited in visual training while the effect on IOP can be of advantage to individuals with rased intraocular pressure in instances where the use of conventional drugs are less efficacious.

\section{References}

1. Glendhill D. West African Trees, Longman Group. Ltd. London 1972.

2. Irvine, FR. Woody Plants of Ghana, Oxford University Press, 1981 pp 145-148.

3. Harley, GN. Nature of African Medicine, Frank Cuss and Co. Ltd., London, 1970.

4. Adefule-Ositelu, AO, Adefule, AK; Dosa BOS, and Onyenafa, PC. Antifungal activities of Garcinia Kolanut extracts on purulent discharges in Lagos University Teaching Hospital, Lagos, Nig. Quarterly J Hospital Medicine 200414 112-114.

5. Cotteril, PJ, Schiemman, F and Stenhouse, AI. Naturally occuring Antisepatics. Journal of Chemical Society $1978 \mathbf{8}$ 532-539.

6. Anyensu, ES. Medicinal plants of West Africa. Publications Inc. Algonac, Michigan, Publications inc. 1978 p. 162.

7. Iwu, MM. Garcinia cola Biflavondoids and forms of treatment for Hepatitis and Hepatoxicity, Nigerian Patent 1982 82 426-481.

8. Ogu, EO. Garcinia cola as a possible substitute to hop in Brewing. M. Sc. Thesis Department of Microbiology, University of Nigeria, Nsukka, 1985.

9. Hussain, RA, Owegby, AG, Parimod, P, and Waterman, RG. Kolanone, a novel polysioprenylated benzophenone with antimicrobial properties from the fruit of Garcinia cola Heckel. Plant Medica 198244 78-81.

10. Bohn K. The Flavonoids, A review of their Physiology, Pharmacodynamics and Therapeutic uses. New York; Aulenof Warth, 1968 pp. 137, 214.

11. Elekwa OK. Prevention of thiacetimide - induced hepatotoxicity by Kolaviron. B. Pharm Thesis. Department of Pharmacology University of Nigeria, Nsukka, 1985.

12. Igboko, AO. The anti-inflammatory biflavonoid and other constituents of Garcinia cola, a Ph.D Thesis Department of Pharmacognosy, University of Nigeria, Nsukka, 1986.

13. Green, DG, Powers, MK. and Banks MS, Depth of focus, eye size and visual acuity. Vision Research 198020 827-835.

14. Iyamu, E. and Ajayi OB. Effect of $G$. cola on the intraocular pressure of young Nigerian adults. Journal of Health \& Visual Sciences 20068 10-4. 